\title{
HOW CAN A SIMPLE PATIENT'S REGISTRY IMPROVE CHRONIC DISEASES CARE?
}

\author{
Soraya Siabani
}

MD, PhD - Assistant Professor at Department of Health Promotion, Public Health School, Kermanshah University of Medical Sciences (KUMS)

\section{Correspondence:}

Dr. Soraya Siabani -Assistant Professor at Department of Health Promotion, Public Health School, Kermanshah University of Medical Sciences (KUMS), Kermanshah, Iran, Registered External Supervisor at the University technology Sydney(UTS), NSW, Australia, Tel: +989183594547, E-mail: ssia5034@uni.sydney.edu.au

\section{TYPE OF ARTICLE: CONFERENCE ABSTRACT}

\begin{abstract}
Introduction: The health ministry of Iran, recently set a goal of developing disease registries and cohort studies. A common question was about the rationale supporting such a decision and policy. In order to achieve a clear understanding and test the hypothesis of "disease registries are associated with the quality of health care management", reviewing literature would be an appropriate strategy. The aim of this study was to explain the association of disease registry and improve disease care with disease registrations.

Methods: The medical electronic databases including PubMed, Ovide, Google Scholar and Science Direct and scientific websites in which the most relevant and valid articles were searched to retrieve relevant articles. Keywords and terms related to disease registry advantages and benefits were used.

Results: The results illustrated that disease registries play a key role in realizing the errors and complications of health care services and improve medical adherence.

Conclusion: We can conclude that disease registries are assisting health care systems to upgrade health care services and increase quality of care.

KEYWORDS: Disease registry, quality of care, medical adherence, care monitoring, health system errors, chronic diseases follow up
\end{abstract}

\footnotetext{
Abstracts of First National Congress of Medical Informatics, Mashhad, Iran, February 2017

(C) 2017 The Authors. This is an open access article under the terms of the Creative Commons Attribution-NonCommercialNoDerivs License, which permits use and distribution in any medium, provided the original work is properly cited, the use is non-commercial and no modifications or adaptations are made.
} 